J. of Inequal. \& Appl., 2001, Vol. 6, pp. 149-165

Reprints available directly from the publisher

Photocopying permitted by license only
(C) 2001 OPA (Overseas Publishers Association) N.V.

Published by license under the Gordon and Breach Science

Publishers imprint.

Printed in Singapore.

\title{
On Bounded Solutions in a Given Set of Systems of Differential Equations
}

\author{
BOHUMIL KRAJC*
}

Department of Applied Mathematics, Faculty of Electrical Engineering and Informatics, Technical University of Ostrava, 17 Listopadu,

70833 Ostrava-Poruba, Czech Republic

(Received 28 August 1999; Revised 10 November 1999)

Entirely bounded solutions in given sets of differential systems are proved sequentially. This enables us to prove several bounded solutions separated in different domains.

Keywords: Asymptotic boundary value problems; Boundedness in a given set; Differential systems

1991 Mathematics Subject Classification: 34B15, 34C11

\section{INTRODUCTION}

Consider the system of ODEs

$$
x^{\prime}=f(t, x) \quad\left({ }^{\prime}=\mathrm{d} / \mathrm{d} t\right)
$$

where $x=\left(x_{1}, \ldots, x_{n}\right)$ and $f=\left(f_{1}, \ldots, f_{n}\right)$ is a continuous vector function which is defined in $\mathbb{R}^{1+n}$. Let $\underline{x}$ be a (classical) solution of $(1.1)$ in $\mathbb{R}$. $\underline{x}$ is said to be entirely bounded if

$$
\sup _{t \in \mathbb{R}}|\underline{x}(t)|<+\infty
$$

Although many papers deal with the existence problem for entirely bounded solutions of differential systems (see e.g. [A1,AGG] and the

*E-mail: bohumil.krajc@vsb.cz. 
references therein), only few results can be used for proving the existence of two or more bounded solutions (see e.g. [A2,AK,K]).

In $[\mathrm{AK}]$ the techniques which consist mainly in invariantness of prescribed sets and transversality arguments on their boundaries were applied to derive multiplicity results. In this paper we develop mentioned method for another class of differential systems. For a comparison with analogous results related to the periodic boundary value problem we refer the reader e.g. to the paper [FZ].

\section{NOTATIONS AND PRELIMINARIES}

2.1. In the whole paper we assume that $\alpha, \beta, \gamma, \delta, l, m, n, o$ are natural numbers and $\varepsilon, \omega$ are positive real numbers.

2.2. By $\{F\}$ we denote a set whose elements satisfy condition $F$. So, we can write $\underline{x} \in\{(1.2)\}$ for an entirely bounded solution $\underline{x}$ of the system (1.1).

The general quantifier will be denoted by $\forall$ and for the existence quantifier we reserve the symbol $\exists$.

2.3. If $\mathcal{M}$ is a subset of some topological space then by $\operatorname{cl} \mathcal{M}$, int $\mathcal{M}$, fr $\mathcal{M}$ we mean the closure, interior, boundary of $\mathcal{M}$, respectively.

2.4. As usual, $\mathbb{R}^{+}=(0,+\infty)$. The standard scalar product between $\xi, \psi \in \mathbb{R}^{n}$ will be denoted by $\xi \cdot \psi$. Furthermore, $|\xi|=\sqrt{\xi \cdot \xi}$, for $\xi \in \mathbb{R}^{n}$.

By $\nabla r$ we denote the gradient of a continuously differentiable real function $r$.

2.5. For a 4-tuple $(\alpha, \beta, \gamma, \delta)$ and $n=\alpha+\beta+\gamma+\delta$, define in the sequel,

$$
\begin{aligned}
S & : \mathbb{R}^{n} \times \mathbb{R} \times\{1, \ldots, n\} \rightarrow \mathbb{R}^{n}, \\
& S(\xi, y, i)=\left(\xi_{1}, \ldots, \xi_{i-1}, y, \xi_{i+1}, \ldots, \xi_{n}\right), \\
P & : \mathbb{R}^{n} \rightarrow \mathbb{R}^{\gamma}, \quad P(\xi)=\left(\xi_{\alpha+\beta+1}, \ldots, \xi_{\alpha+\beta+\gamma}\right), \\
R & : \mathbb{R}^{n} \rightarrow \mathbb{R}^{\delta}, \quad R(\xi)=\left(\xi_{\alpha+\beta+\gamma+1}, \ldots, \xi_{n}\right) .
\end{aligned}
$$

2.6. Let $\mathcal{M} \subset \mathbb{R}^{m}, \mathcal{C}\left(\mathcal{M}, \mathbb{R}^{n}\right)$ be a set of all continuous vector functions defined in $\mathcal{M}$ and with values in $\mathbb{R}^{n}$. If $\mathcal{M}$ is compact we add the norm

$$
\|q\|=\max _{\xi \in \mathcal{M}}|q(\xi)|
$$


to obtain the standard Banach space. If $\mathcal{M}$ is not compact we endow the set $\mathcal{C}\left(\mathcal{M}, \mathbb{R}^{n}\right)$ with a topology of the uniform convergence on compact subsets of $\mathcal{M}$ to make the Fréchet space.

$\mathcal{U} \subset \mathcal{C}\left(\mathcal{M}, \mathbb{R}^{n}\right)$ is said to be globally bounded if

$$
\exists_{\varepsilon \in \mathbb{R}^{+}} \forall_{q \in \mathcal{U}} \sup _{\xi \in \mathcal{M}}|q(\xi)|<\varepsilon .
$$

2.7. Let $\mathcal{U} \subset \mathcal{C}\left(\mathcal{M}, \mathbb{R}^{n}\right)$. For $\mathcal{N} \subset \mathcal{M}$, denote by $\operatorname{re}(\mathcal{U}, \mathcal{N})$ the set

$$
\left\{q \in \mathcal{C}\left(\mathcal{N}, \mathbb{R}^{n}\right): \exists_{p \in \mathcal{U}} \forall_{\xi \in \mathcal{N}} q(\xi)=p(\xi)\right\}
$$

2.8. Let $\mathbb{R}^{n} \supset \mathcal{O} \neq \emptyset$ be a bounded domain such that

$$
\mathcal{O}=\left\{\xi \in \mathbb{R}^{n}: r_{i}(\xi)<0, \text { for } i=1, \ldots, m\right\},
$$

where $r=\left(r_{1}, \ldots, r_{m}\right) \in \mathcal{C}\left(\mathbb{R}^{n}, \mathbb{R}^{m}\right)$ is continuously differentiable. Then we say $\mathcal{O}$ is canonical and write $\mathcal{O}=\operatorname{can}(r)$. Furthermore, for every $\xi \in \operatorname{fr} \mathcal{O}$, set

$$
\mathrm{ca}(\xi, r)=\left\{i: r_{i}(\xi)=0\right\} .
$$

The following lemmas give appropriate estimates for solutions of scalar equations and differential systems.

2.9. LEMMA Consider the 5-tuple $(\underline{x}, f, \omega, u, v)$, where $f \in \mathcal{C}(\mathbb{R} \times \mathbb{R}, \mathbb{R})$, $u, v$ are continuously differentiable functions and $\underline{x}$ is a solution of a scalar equation

$$
x^{\prime}=f(t, x), \quad t \in[-\omega, \omega] .
$$

Suppose that, for every $t \in[-\omega, \omega]$,

$$
\begin{gathered}
u(t)<v(t), \\
f(t, u(t))>u^{\prime}(t), \quad f(t, v(t))<v^{\prime}(t), \\
u(-\omega)<\underline{x}(-\omega)<v(-\omega) .
\end{gathered}
$$

Then $u(t)<\underline{x}(t)<v(t)$, for every $t \in[-\omega, \omega]$. 
Proof We show that $x(t)>u(t)$, for all $t \in[-\omega, \omega]$. The verification of the second inequality can be obtained quite analogously. Assume, contradictionally, the existence of a $t \in(-\omega, \omega]$ such that $\underline{x}(t) \leq u(t)$. Denoting $\tau=\inf \{t \in(-\omega, \omega]: \underline{x}(t) \leq u(t)\}$, we have obviously $\underline{x}(\tau)=u(\tau)$. Setting $p=\underline{x}-u$, the inequality $p^{\prime}(\tau)=f(\tau, u(\tau))-u^{\prime}(\tau)>0$ implies the existence of $\varepsilon \in \mathbb{R}^{+}$such that $p^{\prime}(\sigma)>0$, for every $\sigma \in(\tau-\varepsilon, \tau]$. Taking any $t \in(\tau-\varepsilon, \tau)$, and applying the Lagrange mean value theorem, we get

$$
\begin{aligned}
& \underline{x}(t)-u(t)=p(t)-p(\tau)=p^{\prime}(\sigma)(t-\tau)<0, \\
& \quad \text { for some } \sigma \in(t, \tau) .
\end{aligned}
$$

Hence $\underline{x}(t)<u(t)$, for $t \in(\tau-\varepsilon, \tau)$, which disagrees with the definition of $\tau$.

2.10. Remark Under assumptions of the lemma above, replacing (2.9.4) by

$$
u(-\omega) \leq \underline{x}(-\omega) \leq v(-\omega),
$$

we obtain $u(t)<\underline{x}(t)<v(t)$, for every $t \in(-\omega, \omega]$.

By the same manner, we can derive

2.11. LEMMA Assume that $(\underline{x}, f, \omega, u, v) \in \bigcap_{i=1}^{2}\{(2.9 . i)\}$ and, for every $t \in[-\omega, \omega]$,

$$
\begin{gathered}
f(t, u(t))<u^{\prime}(t), \quad f(t, v(t))>v^{\prime}(t), \\
u(\omega)<\underline{x}(\omega)<v(\omega) .
\end{gathered}
$$

Then the same assertion as in Lemma 2.9 holds for $\underline{x}$.

2.12. Remark Of course, replacing (2.11.2) by

$$
u(\omega) \leq \underline{x}(\omega) \leq v(\omega),
$$

we obtain $u(t)<\underline{x}(t)<v(t)$, for every $t \in[-\omega, \omega)$.

2.13. LeMmA Let $\mathcal{O}=\operatorname{can}(r)$ and $\underline{x}$ be a solution of the differential system

$$
x^{\prime}=f(t, x), \quad t \in[-\omega, \omega],
$$


where $f \in \mathcal{C}\left(\mathbb{R} \times \mathbb{R}^{n}, \mathbb{R}^{n}\right)$. Assume that $(\underline{x}, f, \omega, \mathcal{O})$ satisfies

$$
\begin{gathered}
\forall_{t \in[-\omega, \omega]} \forall_{\xi \in \mathrm{fr} \mathcal{O}} \forall_{i \in \mathrm{ca}(\xi, r)} \quad \nabla r_{i}(\xi) \cdot f(t, \xi)<0, \\
\underline{x}(-\omega) \in \mathcal{O} .
\end{gathered}
$$

Then, for every $t \in[-\omega, \omega], \underline{x}(t) \in \mathcal{O}$.

Proof Assume, contradictionally, the existence of $t \in(-\omega, \omega]$ such that $\underline{x}(t) \notin \mathcal{O}$. Denoting $\tau=\inf \{t \in(-\omega, \omega]: \underline{x}(t) \notin \mathcal{O}\}$, we have obviously $\underline{x}(\tau) \in$ fr $\mathcal{O}$. Hence, $r_{i}(\underline{x}(\tau))=0$ for some $i \in \operatorname{ca}(\underline{x}(\tau), r)$. Choosing such $i$ and setting $p(t)=r_{i}(\underline{x}(t))$, we obtain $p(\tau)=0$ and, for every $t \in[-\omega, \tau)$, $p(t)<0$.

But, at the same time, (2.13.2) implies the existence of $\varepsilon \in \mathbb{R}^{+}$such that the inequality $r_{i}(\underline{x}(t))=p(t)=p(t)-p(\tau)=p^{\prime}(\sigma)(t-\tau)>0$ holds for $t \in[\tau-\varepsilon, \tau)$, which contradicts the definition of $\tau$.

2.14. Remark Instead of (2.13.3) one can assume

$$
\underline{x}(-\omega) \in \operatorname{cl} \mathcal{O},
$$

to obtain $\underline{x}(t) \in \mathcal{O}$, for every $t \in(-\omega, \omega]$.

Quite analogously as in the proof of Lemma 2.13 we can derive

2.15. LEMMA If $(\underline{x}, f, \omega, \mathcal{O}) \in\{(2.13 .1)\}$ and

$$
\begin{gathered}
\forall_{t \in[-\omega, \omega]} \forall_{\xi \in \mathrm{fr} \mathcal{O}} \forall_{i \in \mathrm{ca}(\xi, r)} \quad \nabla r_{i}(\xi) \cdot f(t, \xi)>0, \\
\underline{x}(\omega) \in \mathcal{O},
\end{gathered}
$$

then the same assertion as in the previous lemma is true.

2.16. Remark Replacing (2.15.2) by

$$
\underline{x}(\omega) \in \operatorname{cl} \mathcal{O},
$$

we conclude $\underline{x}(t) \in \mathcal{O}$, for every $t \in[-\omega, \omega)$.

The following lemma represents a slightly improved version in [K]. 
2.17. LEMMA Consider the pair $(f, \mathcal{Q})$, where $f \in \mathcal{C}\left(\mathbb{R} \times \mathbb{R}^{n}, \mathbb{R}^{n}\right)$ and $\mathcal{Q} \subset \mathcal{C}\left(\mathbb{R}, \mathbb{R}^{n}\right)$ is globally bounded. Assume that the problems $(l=1,2, \ldots)$

$$
\begin{aligned}
& x^{\prime}=f(t, x), \quad t \in[-l, l], \\
& x \in \operatorname{re}(\mathcal{Q},[-l, l])
\end{aligned}
$$

admit solutions $\underline{x}_{(l)}$. Then there exists an entirely bounded solution $\underline{x} \in \operatorname{cl} \mathcal{Q}$ of system (1.1).

Proof For abbreviation, let $\left\{\underline{x}_{(l)}\right\}_{l=1}^{+\infty}$ stand for the sequence of appropriate extensions of solutions $\underline{x}_{(l)}$ in $\mathbb{R}$. Due to the well-known Arzelá-Ascoli theorem and the diagonalization arguments, we are able to choose a subsequence $\left\{\underline{x}_{\left(l_{i}\right)}\right\}_{i=1}^{+\infty}$ which converges to a solution $\underline{x} \in \operatorname{cl} \mathcal{Q}$ of the system (1.1) (for more details, see e.g. [K, pp. 178-180]).

To ensure the solvability of problems (2.17.1), we apply the special form of the Leray-Schauder continuation principle (see e.g. [LS]).

2.18. Proposition Let $\mathcal{U} \subset \mathcal{C}\left([-l, l], \mathbb{R}^{n}\right)$ be a nonempty, open and bounded set, $\Lambda: \mathrm{cl} \mathcal{U} \times[0,1] \rightarrow \mathcal{C}\left([-l, l], \mathbb{R}^{n}\right)$. Assume that $(\Lambda, \mathcal{U})$ satisfies

$\Lambda$ is a continuous operator with relatively compact image

$$
\Lambda(\mathrm{cl} \mathcal{U} \times[0,1]),
$$

there exists $q^{*} \in \mathcal{U}$ such that $\Lambda(q, 0)=q^{*}$, for every $q \in \mathrm{cl} \mathcal{U}$,

$$
q \neq \Lambda(q, \lambda), \text { for every } q \in \text { fr } \mathcal{U} \text { and all } \lambda \in[0,1]
$$

Then the equation $x=\Lambda(x, 1)$ admits at least one solution in $\mathcal{U}$.

The last statement of this section follows immediately from the result developed in [CFM].

2.19. PROPOSITION $\quad$ Let $\mathcal{S} \subset \mathcal{C}\left([-l, l], \mathbb{R}^{n}\right)$ be a closed set and $\mathcal{U} \neq \emptyset$ be a bounded subset of $\mathcal{C}\left([-l, l], \mathbb{R}^{n}\right)$. Consider the problems

$$
\begin{aligned}
& x^{\prime}=f(t, q(t), \lambda), \\
& x \in \mathcal{S},
\end{aligned}
$$

where $f \in \mathcal{C}\left(\mathbb{R} \times \mathbb{R}^{n} \times[0,1], \mathbb{R}^{n}\right)$, and suppose that 
for every $(q, \lambda) \in \mathrm{cl} \mathcal{U} \times[0,1],(2.19 .1)$ admits exactly one solution $\Lambda(q, \lambda)$ and $\Lambda(\mathrm{cl} \mathcal{U} \times[0,1])$ is a bounded subset of $\mathcal{C}\left([-l, l], \mathbb{R}^{n}\right)$.

Then $\Lambda$ is a continuous map with relatively compact image.

\section{MAIN RESULTS}

3.1. Consider the 5-tuple $\left(f, u, v, \mathcal{O}_{1}, \mathcal{O}_{2}\right)$, where $f \in \mathcal{C}\left(\mathbb{R} \times \mathbb{R}^{n}, \mathbb{R}^{n}\right)$, $n=\alpha+\beta+\gamma+\delta, \quad \mathbb{R}^{\gamma} \supset \mathcal{O}_{1}=\mathrm{ca}\left(r_{(1)}\right), \quad \mathbb{R}^{\delta} \supset \mathcal{O}_{2}=\mathrm{ca}\left(r_{(2)}\right) . \quad$ Let $u \in \mathcal{C}\left(\mathbb{R}, \mathbb{R}^{\alpha+\beta}\right)$ and $v \in \mathcal{C}\left(\mathbb{R}, \mathbb{R}^{\alpha+\beta}\right)$ be continuously differentiable.

Denote, in the sequel,

$$
\begin{gathered}
\mathcal{Q}_{1}=\left\{q \in \mathcal{C}\left(\mathbb{R}, \mathbb{R}^{n}\right): \forall_{t \in \mathbb{R}} \forall_{i=1, \ldots, \alpha+\beta} u_{i}(t)<q_{i}(t)<v_{i}(t)\right\}, \\
\mathcal{Q}_{2}=\left\{q \in \mathcal{C}\left(\mathbb{R}, \mathbb{R}^{n}\right): \forall_{t \in \mathbb{R}} P(q(t)) \in \mathcal{O}_{1}, R(q(t)) \in \mathcal{O}_{2}\right\}, \\
\mathcal{Q}=\mathcal{Q}_{1} \bigcap \mathcal{Q}_{2}, \\
\mathcal{M}=\left\{\xi \in \mathbb{R}^{n}: \exists_{q \in \mathrm{cl} \mathcal{Q}} \exists_{t \in \mathbb{R}} q(t)=\xi\right\} .
\end{gathered}
$$

THEOREM Suppose that the conditions below hold for every $t \in \mathbb{R}, \xi \in \mathcal{M}$ and $i=1, \ldots, \alpha, j=\alpha+1, \ldots, \alpha+\beta$.

$\mathcal{Q}$ is globally bounded and there exists a constant function $q^{*} \in \mathcal{Q}$,

$$
\begin{array}{ll}
f_{i}\left(t, S\left(\xi, u_{i}(t), i\right)\right)>u_{i}^{\prime}(t), & f_{i}\left(t, S\left(\xi, v_{i}(t), i\right)\right)<v_{i}^{\prime}(t), \\
f_{j}\left(t, S\left(\xi, u_{j}(t), j\right)\right)<u_{j}^{\prime}(t), & f_{j}\left(t, S\left(\xi, v_{j}(t), j\right)\right)>v_{j}^{\prime}(t),
\end{array}
$$

$$
\left(P(\xi) \in \operatorname{fr} \mathcal{O}_{1} \wedge k \in \mathrm{ca}\left(P(\xi), r_{(1)}\right)\right) \Rightarrow \nabla r_{(1) k}(P(\xi)) \cdot P(f(t, \xi))<0,
$$

$$
\left(R(\xi) \in \operatorname{fr} \mathcal{O}_{2} \wedge k \in \mathrm{ca}\left(R(\xi), r_{(2)}\right)\right) \Rightarrow \nabla r_{(2) k}(R(\xi)) \cdot R(f(t, \xi))>0 .
$$

Then (1.1) admits at least one solution $\underline{x} \in \mathcal{Q}$. 
Proof It will be divided into four consecutive steps. Denote

$$
u_{i}^{*}=\inf _{t \in \mathbb{R}} u_{i}(t)-1, \quad v_{i}^{*}=\sup _{t \in \mathbb{R}} v_{i}(t)+1, \quad \text { for } i=1, \ldots, \alpha+\beta .
$$

A. Let $\mathcal{Q}_{1}^{*}=\left\{q \in \mathcal{C}\left(\mathbb{R}, \mathbb{R}^{n}\right): \forall_{t \in \mathbb{R}} \forall_{i=1, \ldots, \alpha+\beta} u_{i}^{*}<q_{i}(t)<v_{i}^{*}\right\}$ and

$$
\mathcal{Q}^{*}=\mathcal{Q}_{1}^{*} \bigcap \mathcal{Q}_{2}
$$

Then there exists a function $g \in \mathcal{C}\left(\mathbb{R} \times \mathbb{R}^{n}, \mathbb{R}^{n}\right)$ with the following properties:

$$
\begin{array}{lll}
\forall_{q \in \mathrm{cl} \mathcal{Q}} & \forall_{t \in \mathbb{R}} & g(t, q(t))=f(t, q(t)), \\
\forall_{q \in \mathrm{cl} \mathcal{Q}^{*}} \forall_{t \in \mathbb{R}} \forall_{i=1, \ldots, \alpha} & g_{i}\left(t, S\left(q(t), u_{i}^{*}, i\right)\right)=1, \\
\forall_{q \in \mathrm{cl} \mathcal{Q}^{*}} \forall_{t \in \mathbb{R}} \forall_{i=1, \ldots, \alpha} & g_{i}\left(t, S\left(q(t), v_{i}^{*}, i\right)\right)=-1, \\
\forall_{q \in \mathrm{cl} \mathcal{Q}^{*}} \forall_{t \in \mathbb{R}} & \forall_{j=\alpha+1, \ldots, \alpha+\beta} & g_{j}\left(t, S\left(q(t), u_{j}^{*}, j\right)\right)=-1, \\
\forall_{q \in \mathrm{cl} \mathcal{Q}^{*}} \forall_{t \in \mathbb{R}} \forall_{j=\alpha+1, \ldots, \alpha+\beta} & g_{j}\left(t, S\left(q(t), v_{j}^{*}, j\right)\right)=1 .
\end{array}
$$

To assure the statement above, define, for $k=1, \ldots, \alpha+\beta, y \in \mathbb{R}$, $x \in \mathbb{R}^{n}$,

$$
\begin{gathered}
\eta_{k}(t, y)= \begin{cases}u_{k}(t) & \text { for } y \in\left(-\infty, u_{k}(t)\right), \\
y & \text { for } y \in\left[u_{k}(t), v_{k}(t)\right], \\
v_{k}(t) & \text { for } y \in\left(v_{k}(t),+\infty\right),\end{cases} \\
\chi(t, x)=\left(\eta_{1}\left(t, x_{1}\right), \ldots, \eta_{\alpha+\beta}\left(t, x_{\alpha+\beta}\right), P(x), R(x)\right) .
\end{gathered}
$$

Then components of the founded function $g$ can take the form

$$
g_{i}(t, x)= \begin{cases}\frac{f_{i}(t, \chi(t, x))-1}{u_{i}(t)-u_{i}^{*}}\left(x_{i}-u_{i}^{*}\right)+1 & \text { for } x_{i} \in\left(-\infty, u_{i}(t)\right), \\ f_{i}(t, \chi(t, x)) & \text { for } x_{i} \in\left[u_{i}(t), v_{i}(t)\right], \\ \frac{f_{i}(t, \chi(t, x))+1}{v_{i}(t)-v_{i}^{*}}\left(x_{i}-v_{i}^{*}\right)-1 & \text { for } x_{i} \in\left(v_{i}(t),+\infty\right),\end{cases}
$$

provided $i \in\{1, \ldots, \alpha\}$,

$$
g_{j}(t, x)= \begin{cases}\frac{f_{j}(t, \chi(t, x))+1}{u_{j}(t)-u_{j}^{*}}\left(x_{j}-u_{j}^{*}\right)-1 & \text { for } x_{j} \in\left(-\infty, u_{j}(t)\right), \\ f_{j}(t, \chi(t, x)) & \text { for } x_{j} \in\left[u_{j}(t), v_{j}(t)\right], \\ \frac{f_{j}(t, \chi(t, x))-1}{v_{j}(t)-v_{j}^{*}}\left(x_{j}-v_{j}^{*}\right)+1 & \text { for } x_{j} \in\left(v_{j}(t),+\infty\right),\end{cases}
$$


provided $j \in\{\alpha+1, \ldots, \alpha+\beta\}$,

$$
P(g(t, x))=P(f(t, \chi(t, x))), \quad R(g(t, x))=R(f(t, \chi(t, x))) .
$$

B. Let $l$ be a fixed natural number and $\mathcal{U}=\operatorname{re}\left(\mathcal{Q}^{*},[-l, l]\right)$. Then the system

$$
x^{\prime}=g(t, x), \quad t \in[-l, l]
$$

admits a solution $\underline{x}_{(l)} \in \mathcal{U}$.

To prove this part consider a class of problems

$$
\begin{aligned}
& x^{\prime}=\lambda g(t, q(t)), \quad q \in \mathrm{cl} \mathcal{U}, t \in[-l, l], \\
& \forall_{i=1, \ldots, \alpha} x_{i}(-l)=q_{i}^{*}, \quad \forall \forall_{j=\alpha+1, \ldots, \alpha+\beta} x_{j}(l)=q_{j}^{*}, \\
& P(x(-l))=P\left(q^{*}\right), \quad R(x(l))=R\left(q^{*}\right) .
\end{aligned}
$$

Define the operator $\Lambda: \mathrm{cl} \mathcal{U} \times[0,1] \rightarrow \mathcal{C}\left([-l, l], \mathbb{R}^{n}\right)$, which assigns, to any pair $(q, \lambda) \in \operatorname{cl} \mathcal{U} \times[0,1]$, the unique solution $x_{q, \lambda}=\Lambda(q, \lambda)$ of the related problem in (3.1.7). Clearly, any fixed point of the operator $\Lambda(\cdot, 1)$ represents a solution of (3.1.6). Denoting by $\mathcal{S}$ a set of all functions in $\mathcal{C}\left([-l, l], \mathbb{R}^{n}\right)$ which satisfy the boundary conditions in (3.1.7) we see that $(\lambda g, \mathcal{U}, \mathcal{S})$ satisfies assumptions of Proposition 2.19. Hence, $\Lambda$ is a continuous map with a relatively compact image. Evidently, (2.18.2) holds for $(\Lambda, \mathcal{U})$ too. Since $\mathcal{U}$ is open we confirm the validity of the part of (2.18.3) related to $\lambda=0$. So, it remains to prove

$$
\forall_{q \in \mathrm{fr} \mathcal{U}} \forall_{\lambda \in(0,1]} \quad q \neq \Lambda(q, \lambda) .
$$

Assume, contradictionally, the existence of $(q, \lambda) \in \operatorname{fr} \mathcal{U} \times(0,1]$ such that $\underline{q}=\Lambda(\underline{q}, \lambda)$. Let $\tau \in[-l, l]$ be a number for which $\underline{q}(\tau) \in$ $\operatorname{re}(\operatorname{fr} \mathcal{U},\{\tau\})$.

Define

$$
\begin{aligned}
& \forall_{k=1, \ldots, \alpha+\beta}\left(h_{k} \in \mathcal{C}(\mathbb{R} \times \mathbb{R}, \mathbb{R}), h_{k}(t, x)=\lambda g_{k}(t, S(\underline{q}(t), x, k))\right), \\
& h_{P} \in \mathcal{C}\left(\mathbb{R} \times \mathbb{R}^{\gamma}, \mathbb{R}^{\gamma}\right), \\
& h_{P}(t, x)=P\left(\lambda g\left(t, \underline{q}_{1}(t), \ldots, \underline{q}_{\alpha+\beta}(t), x, \underline{q}_{\alpha+\beta+\gamma+1}(t), \ldots, \underline{q}_{n}(t)\right)\right), \\
& h_{R} \in \mathcal{C}\left(\mathbb{R} \times \mathbb{R}^{\delta}, \mathbb{R}^{\delta}\right), h_{R}(t, x)=R\left(\lambda g\left(t, \underline{q}_{1}(t), \ldots, \underline{q}_{\alpha+\beta+\gamma}(t), x\right)\right) .
\end{aligned}
$$


Then, for $i=1, \ldots, \alpha$ and $j=\alpha+1, \ldots, \alpha+\beta$,

$$
\begin{aligned}
& \left(\underline{q}_{i}, h_{i}, l, u_{i}^{*}, v_{i}^{*}\right) \in \bigcap_{k=1}^{4}\{(2.9 . k)\}, \\
& \left(\underline{q}_{j}, h_{j}, l, u_{j}^{*}, v_{j}^{*}\right) \in\left(\bigcap_{k=1}^{2}\{(2.9 . k)\}\right) \bigcap\left(\bigcap_{k=1}^{2}\{(2.11 . k)\}\right), \\
& \left(P(\underline{q}), h_{P}, l, \mathcal{O}_{1}\right) \in \bigcap_{k=1}^{3}\{(2.13 . k)\}, \\
& \left(R(\underline{q}), h_{R}, l, \mathcal{O}_{2}\right) \in \bigcap_{k=1}^{2}\{(2.15 . k)\} \bigcap\{(2.13 .1)\} .
\end{aligned}
$$

Hence, applying Lemmas 2.9, 2.11, 2.13 and 2.15, respectively, we obtain $q(\tau) \in \operatorname{re}(\mathcal{U},\{\tau\})$ which contradicts the characteristic property of $\tau$.

Since all assumptions of Proposition 2.18 are fulfilled, the existence of a solution $\underline{x}_{(l)} \in \mathcal{U}$ is ensured.

C. Recall that $g(t, q(t))=f(t, q(t))$, for every $q \in \mathrm{cl} \mathcal{Q}$ and all $t \in \mathbb{R}$. So, repeating definition (3.1.8) with $\lambda=1$ and $\underline{q}=\underline{x}_{(l)}$, we can apply conditions (3.1.2), (3.1.3) to obtain $\underline{x}_{(l)} \in \operatorname{re}(\mathcal{Q},[-l, l])$. Since $l \in \mathbb{N}$ was chosen arbitrarily $(f, \mathcal{Q})$ satisfies assumptions of Lemma 2.17 and we conclude that

(1.1) admits a solution $\underline{x} \in \operatorname{cl} \mathcal{Q}$.

To finish the proof it is sufficient to confirm

D. If there exists a solution $\underline{x} \in \operatorname{cl} \mathcal{Q}$ of $(1.1)$ then $\underline{x} \in \mathcal{Q}$.

Assume, for a contradiction, the existence of $\tau \in \mathbb{R}$ such that

$$
\underline{x}(\tau) \in \operatorname{re}(\operatorname{cl} \mathcal{Q},\{\tau\}) \backslash \operatorname{re}(\mathcal{Q},\{\tau\}) .
$$

Let $\omega=|\tau|+1$. Define, in the sequel,

$$
\begin{aligned}
& \forall_{k=1, \ldots, \alpha+\beta}\left(h_{k} \in \mathcal{C}(\mathbb{R} \times \mathbb{R}, \mathbb{R}), \quad h_{k}(t, x)=f_{k}(t, S(\underline{x}(t), x, k))\right), \\
& h_{P} \in \mathcal{C}\left(\mathbb{R} \times \mathbb{R}^{\gamma}, \mathbb{R}^{\gamma}\right), \\
& h_{P}(t, x)=P\left(f\left(t, \underline{x}_{1}(t), \ldots, \underline{x}_{\alpha+\beta}(t), x, \underline{x}_{\alpha+\beta+\gamma+1}(t), \ldots, \underline{x}_{n}(t)\right)\right), \\
& h_{R} \in \mathcal{C}\left(\mathbb{R} \times \mathbb{R}^{\delta}, \mathbb{R}^{\delta}\right), \quad h_{R}(t, x)=R\left(f\left(t, \underline{x}_{1}(t), \ldots, \underline{x}_{\alpha+\beta+\gamma}(t), x\right)\right) .
\end{aligned}
$$


Then we conclude, for $i=1, \ldots, \alpha$ and $j=\alpha+1, \ldots, \alpha+\beta$,

$$
\begin{aligned}
& \left(\underline{x}_{i}, h_{i}, \omega, u_{i}, v_{i}\right) \in \bigcap_{k=1}^{3}\{(2.9 . k)\} \bigcap\{(2.10 .1)\}, \\
& \left(\underline{x}_{j}, h_{j}, \omega, u_{j}, v_{j}\right) \in \bigcap_{k=1}^{2}\{(2.9 . k)\} \bigcap\{(2.11 .1)\} \bigcap\{(2.12 .1)\}, \\
& \left(P(\underline{x}), h_{P}, \omega, \mathcal{O}_{1}\right) \in \bigcap_{k=1}^{2}\{(2.13 . k)\} \bigcap\{(2.14 .1)\}, \\
& \left(R(\underline{x}), h_{R}, \omega, \mathcal{O}_{2}\right) \in\{(2.13 .1)\} \bigcap\{(2.15 .1)\} \bigcap\{(2.16 .1)\}
\end{aligned}
$$

Hence, applying Remarks 2.10, 2.12, 2.14 and 2.16, we obtain $\underline{x}(\tau) \in \operatorname{re}(\mathcal{Q},\{\tau\})$ which contradicts the assumption above.

3.2. Evidently, results similar to the theorem above can be derived if one or more constant in $\{\alpha, \beta, \gamma, \delta\}$ vanish. For example, let $\alpha=1$, $\beta=\gamma=\delta=0$ and (1.1) takes a form

$$
x^{\prime}=a(t) x+g(t, x)
$$

where $a \in \mathcal{C}(\mathbb{R}, \mathbb{R}), g \in \mathcal{C}(\mathbb{R} \times \mathbb{R}, \mathbb{R})$. Assume the existence of $C \in \mathbb{R}^{+}$, $\mathbb{R} \ni K<L \in \mathbb{R}$ such that $K \mathrm{e}^{C \operatorname{sgn}(K)}<L \mathrm{e}^{-C \operatorname{sgn}(L)}$ and

$$
\left|\int_{0}^{t} a(s) \mathrm{d} s\right|<C, \quad g\left(t, K \mathrm{e}^{\int_{0}^{t} a(s) \mathrm{d} s}\right)>0, \quad g\left(t, L \mathrm{e}^{\int_{0}^{t} a(s) \mathrm{d} s}\right)<0
$$

hold for every $t \in \mathbb{R}$. Fix $q^{*}=\frac{1}{2}\left(K \mathrm{e}^{C \operatorname{sgn}(K)}+L \mathrm{e}^{-C \operatorname{sgn}(L)}\right)$. Then, letting $u(t)=K \mathrm{e}^{\int_{0}^{t} a(s) \mathrm{d} s}, v(t)=L \mathrm{e}^{\int_{0}^{t} a(s) \mathrm{d} s}$, we obtain

$$
\begin{aligned}
& a(t) u(t)+g(t, u(t))>u^{\prime}(t), \quad a(t) v(t)+g(t, v(t))<v^{\prime}(t), \\
& u(t)<q^{*}<v(t),
\end{aligned}
$$

for all $t \in \mathbb{R}$, hence, (3.2.1) has an entirely bounded solution.

The described technique can be applied to the system

$$
\begin{aligned}
& x_{i}^{\prime}=a_{i}(t) x_{i}+g_{i}(t, x), \quad i=1, \ldots, \alpha+\beta, \\
& x_{j}^{\prime}=g_{j}(t, x), \quad j=\alpha+\beta+1, \ldots, \alpha+\beta+\varepsilon+\omega,
\end{aligned}
$$


where $x=\left(x_{1}, \ldots, x_{n}\right), n=\alpha+\beta+\varepsilon+\omega$ and all functions on the righthand side of the system are continuous. Omitting an easy proof, let us illustrate one of the possible results.

COROLlary System (3.2.2) admits at least $3^{\varepsilon+\omega}$ entirely bounded solutions providing there exist positive real constants $C, D<E$ such that

$$
\begin{gathered}
\forall_{i=1, \ldots, \alpha} \quad \forall_{j=\alpha+1, \ldots, \alpha+\beta} \quad \forall_{k=\alpha+\beta+1, \ldots, \alpha+\beta+\varepsilon} \\
\forall_{l=\alpha+\beta+\varepsilon+1, \ldots, n} \quad \forall_{t \in \mathbb{R}} \quad \forall_{C \mathrm{e}^{-C}<y<C \mathrm{e}^{C}} \\
\left|\int_{0}^{t} a_{i}(s) \mathrm{d} s\right|<C, \quad\left|\int_{0}^{t} a_{j}(s) \mathrm{d} s\right|<C, \\
g_{i}(t, S(\xi,-y, i))>0, \quad g_{i}(t, S(\xi, y, i))<0, \\
g_{j}(t, S(\xi,-y, j))<0, \quad g_{j}(t, S(\xi, y, j))>0, \\
g_{k}(t, S(\xi,-D, k))>0, \quad g_{k}(t, S(\xi, D, k))<0, \\
g_{k}(t, S(\xi,-E, k))<0, \quad g_{k}(t, S(\xi, E, k))>0, \\
g_{l}(t, S(\xi,-D, l))<0, \quad g_{l}(t, S(\xi, D, l))>0, \\
g_{l}(t, S(\xi,-E, l))>0, \quad g_{l}(t, S(\xi, E, l))<0
\end{gathered}
$$

hold for every

$$
\begin{aligned}
& \xi \in\left\{\zeta \in \mathbb{R}^{n}:\left|\zeta_{i}\right| \leq C \mathrm{e}^{C},\left|\zeta_{j}\right| \leq E,\right. \\
& \quad \text { for } i=1, \ldots, \alpha+\beta, j=\alpha+\beta+1, \ldots, n\} .
\end{aligned}
$$

3.3. Set $\alpha=0, \beta=0$ and consider (1.1) in a form

$$
\begin{aligned}
& y^{\prime}=e(t, y, z)+f(t, y, z), \\
& z^{\prime}=g(t, y, z)+h(t, y, z),
\end{aligned}
$$

where $e, f$ are elements in $\mathcal{C}\left(\mathbb{R} \times \mathbb{R}^{\gamma+\delta}, \mathbb{R}^{\gamma}\right)$ and $g, h$ belong to $\mathcal{C}\left(\mathbb{R} \times \mathbb{R}^{\gamma+\delta}, \mathbb{R}^{\delta}\right)$. Assuming a continuity of matrices $\partial e / \partial y, \partial g / \partial z$ let us denote by $\psi(t, y, z)$ the greatest eigenvalue of a symmetrical matrix

$$
\frac{1}{2}\left(\frac{\partial}{\partial y} e(t, y, z)+\left(\frac{\partial}{\partial y} e(t, y, z)\right)^{\top}\right)
$$

and by $\varphi(t, y, z)$ the smallest eigenvalue of

$$
\frac{1}{2}\left(\frac{\partial}{\partial z} g(t, y, z)+\left(\frac{\partial}{\partial z} g(t, y, z)\right)^{\top}\right) .
$$


Recall (see e.g. [D, pp. 284]) that, for any fixed $(t, y, z) \in \mathbb{R}^{1+\gamma+\delta}$ and for every $\xi \in \mathbb{R}^{\gamma}, \eta \in \mathbb{R}^{\delta}$,

$$
\begin{aligned}
& \xi \cdot(e(t, y+\xi, z)-e(t, y, z)) \leq \sup _{\tau \in[0,1]} \psi(t, y+\tau \xi, z)|\xi|^{2}, \\
& \eta \cdot(g(t, y, z+\eta)-g(t, y, z)) \geq \inf _{\tau \in[0,1]} \varphi(t, y, z+\tau \eta)|\eta|^{2} .
\end{aligned}
$$

\section{COROLlaRY Suppose that}

$$
\begin{aligned}
& \sup \left\{\psi(t, \xi, \eta): t \in \mathbb{R}, \xi \in \mathbb{R}^{\gamma}, \eta \in \mathbb{R}^{\delta}\right\} \leq \psi^{*}<0, \\
& \inf \left\{\varphi(t, \xi, \eta): t \in \mathbb{R}, \xi \in \mathbb{R}^{\gamma}, \eta \in \mathbb{R}^{\delta}\right\} \geq \varphi^{*}>0, \\
& \sup \left\{|e(t, 0, \eta)|: t \in \mathbb{R}, \eta \in \mathbb{R}^{\delta}\right\}=E<+\infty, \\
& \sup \left\{|g(t, \xi, 0)|: t \in \mathbb{R}, \xi \in \mathbb{R}^{\gamma}\right\}=G<+\infty, \\
& \limsup _{|\xi| \rightarrow+\infty} \frac{|f(t, \xi, \eta)|}{|\xi|}=F<\left|\psi^{*}\right| \text {, uniformly w.r.t. } t \in \mathbb{R}, \eta \in \mathbb{R}^{\delta}, \\
& \limsup _{|\eta| \rightarrow+\infty} \frac{|h(t, \xi, \eta)|}{|\eta|}=H<\varphi^{*}, \text { uniformly w.r.t. } t \in \mathbb{R}, \xi \in \mathbb{R}^{\gamma} .
\end{aligned}
$$

Then (3.3.1) admits at least one entirely bounded solution.

Proof Fix $\varepsilon \in \mathbb{R}^{+}$such that

$$
\begin{gathered}
\forall_{t \in \mathbb{R}} \forall_{y \in \mathbb{R}^{\gamma}} \forall_{\xi \in \mathbb{R}^{\gamma},|\xi|=\sqrt{2 \varepsilon}} \forall_{z \in \mathbb{R}^{\delta}} \forall_{\eta \in \mathbb{R}^{\delta},|\eta|=\sqrt{2 \varepsilon}} \\
|f(t, \xi, z)|<\frac{1}{2}\left(F+\left|\psi^{*}\right|\right)|\xi|, \quad|h(t, y, \eta)|<\frac{1}{2}\left(H+\varphi^{*}\right)|\eta|, \\
\psi^{*}|\xi|^{2}+E|\xi|+\frac{1}{2}\left(F+\left|\psi^{*}\right|\right)|\xi|^{2}<0, \\
\varphi^{*}|\eta|^{2}-G|\eta|-\frac{1}{2}\left(H+\varphi^{*}\right)|\eta|^{2}>0
\end{gathered}
$$

and define, in the sequel,

$$
\begin{aligned}
r_{(1)} \in \mathcal{C}\left(\mathbb{R}^{\gamma}, \mathbb{R}\right), \quad r_{(1)}(\xi)=\frac{1}{2}|\xi|^{2}-\varepsilon, \\
r_{(2)} \in \mathcal{C}\left(\mathbb{R}^{\delta}, \mathbb{R}\right), \quad r_{(2)}(\eta)=\frac{1}{2}|\eta|^{2}-\varepsilon, \\
\mathcal{O}_{1}=\left\{\zeta \in \mathbb{R}^{\gamma}: r_{(1)}(\zeta)<0\right\}, \quad \mathcal{O}_{2}=\left\{\zeta \in \mathbb{R}^{\delta}: r_{(2)}(\zeta)<0\right\}, \\
\mathcal{Q}=\left\{q \in \mathcal{C}\left(\mathbb{R}, \mathbb{R}^{\gamma+\delta}\right): P(q(t)) \in \mathcal{O}_{1}, R(q(t)) \in \mathcal{O}_{2},\right. \\
\quad \text { for every } t \in \mathbb{R}\} .
\end{aligned}
$$


Clearly, $\mathcal{Q}$ is globally bounded and the constant function 0 belongs to $\mathcal{Q}$. Since

$$
\begin{aligned}
& \nabla r_{(1)}(\xi) \cdot(e(t, \xi, z)+f(t, \xi, z)) \\
& \quad=\xi \cdot(e(t, \xi, z)-e(t, 0, z))+\xi \cdot e(t, 0, z)+\xi \cdot f(t, \xi, z),
\end{aligned}
$$

we get, applying the Cauchy inequality and assumptions above,

$$
\nabla r_{(1)}(\xi) \cdot(e(t, \xi, z)+f(t, \xi, z)) \leq \psi^{*}|\xi|^{2}+E|\xi|+\frac{1}{2}\left(F+\left|\psi^{*}\right|\right)|\xi|^{2}<0,
$$

for all $\xi \in \mathbb{R}^{\gamma},|\xi|=\sqrt{2 \varepsilon}, z \in \mathbb{R}^{\delta}$. Analogously, we obtain

$$
\nabla r_{(2)}(\eta) \cdot(g(t, y, \eta)+h(t, y, \eta)) \geq \varphi^{*}|\eta|^{2}-G|\eta|-\frac{1}{2}\left(H+\varphi^{*}\right)|\eta|^{2}>0,
$$

for all $y \in \mathbb{R}^{\gamma}, \eta \in \mathbb{R}^{\delta},|\eta|=\sqrt{2 \varepsilon}$, hence our system satisfies (3.1.4), (3.1.5), which completes the proof.

3.4. The sharp inequalities in assumptions of the previous theorem can be weakened.

THEOREM Using the notation in 3.1 suppose that $\mathcal{Q}$ satisfies (3.1.1). Assume the existence of vector fields $s_{(1)} \in \mathcal{C}\left(\mathbb{R}^{\gamma}, \mathbb{R}^{\gamma}\right), s_{(2)} \in \mathcal{C}\left(\mathbb{R}^{\delta}, \mathbb{R}^{\delta}\right)$ such that

$$
\begin{array}{lll}
\forall_{\xi \in \mathrm{fr} \mathcal{O}_{1}} \quad \forall_{k \in \mathrm{ca}\left(\xi, r_{(1)}\right)} & \nabla r_{(1) k}(\xi) \cdot s_{(1)}(\xi)<0, \\
\forall_{\xi \in \mathrm{fr} \mathcal{O}_{2}} \quad \forall_{k \in \mathrm{ca}\left(\xi, r_{(2)}\right)} & \nabla r_{(2) k}(\xi) \cdot s_{(2)}(\xi)>0 .
\end{array}
$$

Let $\left(f, u, v, \mathcal{O}_{1}, \mathcal{O}_{2}\right)$ satisfies, for every $t \in \mathbb{R}, \xi \in \mathcal{M}$ and $i=1, \ldots, \alpha$, $j=\alpha+1, \ldots, \alpha+\beta$, the conditions below:

$$
\begin{aligned}
f_{i}\left(t, S\left(\xi, u_{i}(t), i\right)\right) & \geq u_{i}^{\prime}(t), \quad f_{i}\left(t, S\left(\xi, v_{i}(t), i\right)\right) \leq v_{i}^{\prime}(t), \\
f_{j}\left(t, S\left(\xi, u_{j}(t), j\right)\right) & \leq u_{j}^{\prime}(t), \quad f_{j}\left(t, S\left(\xi, v_{j}(t), j\right)\right) \geq v_{j}^{\prime}(t), \\
(P(\xi) & \left.\in \operatorname{fr} \mathcal{O}_{1} \wedge k \in \mathrm{ca}\left(P(\xi), r_{(1)}\right)\right) \\
\Rightarrow & \nabla r_{(1) k}(P(\xi)) \cdot P(f(t, \xi)) \leq 0 \\
(R(\xi) & \left.\in \operatorname{fr} \mathcal{O}_{2} \wedge k \in \operatorname{ca}\left(R(\xi), r_{(2)}\right)\right) \\
\Rightarrow & \nabla r_{(2) k}(R(\xi)) \cdot R(f(t, \xi)) \geq 0 .
\end{aligned}
$$

Then (1.1) admits at least one solution $\underline{x} \in \operatorname{cl} \mathcal{Q}$. 
Proof Define a function $e \in \mathcal{C}\left(\mathbb{R}^{n}, \mathbb{R}^{n}\right)$ by formulas

$$
\begin{aligned}
& \forall_{i=1, \ldots, \alpha} \forall_{\xi \in \mathbb{R}^{n}} \quad e_{i}(\xi)=q_{i}^{*}-\xi_{i}, \\
& \forall_{j=\alpha+1, \ldots, \alpha+\beta} \forall_{\xi \in \mathbb{R}^{n}} \quad e_{j}(\xi)=\xi_{j}-q_{j}^{*}, \\
& \forall_{\xi \in \mathbb{R}^{n}} P(e(\xi))=s_{(1)}(P(\xi)), \quad R(e(\xi))=s_{(2)}(R(\xi)) .
\end{aligned}
$$

It is easy to see that, for every fixed $m \in \mathbb{N}$, a 5-tuple $(f+(e / m), u, v$, $\left.\mathcal{O}_{1}, \mathcal{O}_{2}\right)$ satisfies assumptions of Theorem 3.1. Consider the related sequence $\left\{\underline{x}_{(m)}\right\}_{m=1}^{+\infty}$ of solutions. Evidently, for every fixed $l \in \mathbb{N}$ and every $m$, equality

$\underline{x}_{(m, l)}(t)=\underline{x}_{(m, l)}(0)+\int_{0}^{t} f\left(s, \underline{x}_{(m, l)}(s)\right)+\frac{1}{m} e\left(\underline{x}_{(m, l)}(s)\right) \mathrm{d} s, \quad t \in[-l, l]$

holds for the restriction $\underline{x}_{(m, l)}$ of $\underline{x}_{(m)}$ in a compact interval $[-l, l]$.

Since $\left\{\underline{x}_{(m, l)} \in \operatorname{re}(\mathcal{Q},[-l, l]): m \in \mathbb{N}\right\}$ is a uniformly bounded and equicontinuous set there exists a subsequence $\left\{\underline{x}_{\left(m_{k}, l\right)}\right\}_{k=1}^{+\infty}$ such that $\lim _{k \rightarrow+\infty} \underline{x}_{\left(m_{k}, l\right)}=\underline{x}_{(l)}$, for some $\underline{x}_{(l)} \in \operatorname{re}(\operatorname{cl} \mathcal{Q},[-l, l])$. Making limit process in (3.4.7) we obtain $\underline{x}_{(l)}(t)=\underline{x}_{(l)}(0)+\int_{0}^{t} f\left(s, \underline{x}_{(l)}(s)\right) \mathrm{d} s$, $t \in[-l, l]$ and, consequently, $\underline{x}_{(l)}$ is a solution of the problem in (2.17.1). Now, the required assertion follows immediately from Lemma 2.17 applied on the pair $(f, \mathrm{cl} \mathcal{Q})$.

3.5. Remark Evidently, if $\mathcal{O}_{1}, \mathcal{O}_{2}$ satisfy (3.1.4), (3.1.5), respectively then

$$
\begin{array}{ll}
\forall_{\xi \in \mathrm{fr} \mathcal{O}_{1}} \quad \forall_{k \in \mathrm{ca}\left(\xi, r_{(1)}\right)} & \nabla r_{(1)}(\xi) \neq 0, \\
\forall_{\xi \in \mathrm{fr} \mathcal{O}_{2}} \quad \forall_{k \in \mathrm{ca}\left(\xi, r_{(2)}\right)} \quad \nabla r_{(2)}(\xi) \neq 0 .
\end{array}
$$

Hence, $\mathcal{O}_{1}$ and $\mathcal{O}_{2}$ have the Lipschitz boundaries. On the contrary, let (3.5.1) hold for $\mathcal{O}_{1}$ and $\mathcal{O}_{2}$. Moreover, suppose that these sets have the Lipschitz boundaries. Then (3.4.1) and (3.4.2) hold for some vector fields $s_{(1)}, s_{(2)}$. 
Therefore we can see that conditions (3.4.1) and (3.4.2) do not mean any restriction on $\mathcal{O}_{1}, \mathcal{O}_{2}$ in comparison with the assumptions of Theorem 3.1.

3.6. We finish with an application of our approach to the existence problem for an entirely bounded solution whose some components are nonnegative, while the others are nonpositive. Consider the system

$$
\begin{aligned}
& x^{\prime}=g(t, x, y), \\
& y^{\prime}=h(t, x, y),
\end{aligned}
$$

where $n=\gamma+\delta, g \in \mathcal{C}\left(\mathbb{R} \times \mathbb{R}^{n}, \mathbb{R}^{\gamma}\right), h \in \mathcal{C}\left(\mathbb{R} \times \mathbb{R}^{n}, \mathbb{R}^{\delta}\right)$. Let $A, B, C, D$ be positive real numbers, $A<B, C<D$. Define, for $i=1, \ldots, \gamma, j=1, \ldots, \delta$, $\xi \in \mathbb{R}^{\gamma}, \zeta \in \mathbb{R}^{\delta}$

$$
\begin{aligned}
& r_{(1) i}(\xi)=-\xi_{i}, \quad r_{(2) j}(\zeta)=\zeta_{j}, \\
& r_{(1) \gamma+1}(\xi)=A-\frac{1}{2}|\xi|^{2}, \quad r_{(2) \delta+1}(\zeta)=C-\frac{1}{2}|\zeta|^{2}, \\
& r_{(1) \gamma+2}(\xi)=\frac{1}{2}|\xi|^{2}-B, \quad r_{(2) \delta+2}(\zeta)=\frac{1}{2}|\zeta|^{2}-D, \\
& r_{(1)}=\left(r_{(1) 1}, \ldots, r_{(1) \gamma+2}\right), \quad r_{(2)}=\left(r_{(2) 1}, \ldots, r_{(2) \delta+2}\right), \\
& \mathcal{O}_{1}=\operatorname{can}\left(r_{(1)}\right), \quad \mathcal{O}_{2}=\operatorname{can}\left(r_{(2)}\right),
\end{aligned}
$$

Recall, (3.5.1) holds for $\mathcal{O}_{1}, \mathcal{O}_{2}$. Hence, under the remark above, we confirm the existence of some vector fields $s_{(1)}, s_{(2)}$ such that $\mathcal{O}_{1}$ and $\mathcal{O}_{2}$ satisfy (3.4.1) and (3.4.2), respectively.

Now a natural modification of Theorem 3.4, related to the choice $\alpha=0, \beta=0$, leads us, immediately, to the verification of the following:

Corollary Suppose that, for $i=1, \ldots, \gamma, j=1, \ldots, \delta$ and all $t \in \mathbb{R}$, conditions

$$
\begin{aligned}
& g_{i}(t, S((\xi, \zeta), 0, i)) \geq 0, \quad h_{j}(t, S((\xi, \zeta), 0, \gamma+j)) \geq 0, \\
& |\xi|=\sqrt{2 A} \Rightarrow \xi \cdot g(t, \xi, \zeta) \geq 0, \quad|\xi|=\sqrt{2 B} \Rightarrow \xi \cdot g(t, \xi, \zeta) \leq 0 \\
& |\zeta|=\sqrt{2 C} \Rightarrow \zeta \cdot h(t, \xi, \zeta) \leq 0, \quad|\zeta|=\sqrt{2 D} \Rightarrow \zeta \cdot h(t, \xi, \zeta) \geq 0
\end{aligned}
$$

hold for every $\xi \in \mathrm{cl} \mathcal{O}_{1}$ and every $\zeta \in \mathrm{cl} \mathcal{O}_{2}$. Then there exists at least one entirely bounded solution $(\underline{x}, \underline{y})$ of the system (3.6.1). Moreover, $\underline{x}(t) \in \operatorname{cl} \mathcal{O}_{1}, \underline{y}(t) \in \mathrm{clO}_{2}$, for every $t \in \mathbb{R}$. 


\section{Acknowledgements}

The author wishes to express his thanks to Prof. J. Andres for suggesting the problem and for many stimulating conversations. This work was supported by project CEZ:J17/98:2724019 of MŠMT.

\section{References}

[A1] J. Andres, Almost-periodic and bounded solutions of Carathéodory differential inclusions, Diff. Int. Eqns., 12 (1999).

[A2] J. Andres, Multiple bounded solutions of differential inclusions: the Nielsen theory approach, J. Diff. Eqns., 155 (1999), 285-310.

[AGG] J. Andres, G. Gabor and L. Gorniewicz, Boundary value problems on infinite intervals, Trans. Amer. Math. Soc., 351(12) (1999), 4861-4903.

[AK] J. Andres and B. Krajc, Bounded solutions in a given set of differential systems, J. Comput. Appl. Math., 113(1-2) (1999), 73-82.

[CFM] M. Cecchi, M. Furi and M. Marini, On continuity and compactness of some nonlinear operators associated with differential equations in non-compact intervals, Nonlin. Anal., T.M.A., 9(2) (1985), 171-180.

[D] B.P. Demidovitch, Lectures on the Mathematical Stability Theory, Nauka, Moscow, 1967. (Russian)

[FZ] M.L.C. Fernandez and F. Zanolin, On periodic solutions, in a given set, for differential systems, Riv. Mat. Pura Appl., 8 (1991), 107-130.

[K] M.A. Krasnosel'skii, The Operator of Translation along the Trajectories of Differential Equations, Nauka, Moscow, 1966. (Russian)

[LS] J. Leray and J. Schauder, Topologie et équations fonctionnelles, Ann. Sci. Ec. Norm. Sup., 51 (1934), 45-78. 